山्山 FRANÇAISE

$>\mathrm{DE}$

필 PÉDAGOGIE

\section{Revue française de pédagogie}

Recherches en éducation

156 | juillet-septembre 2006

Les espaces locaux d'interdépendance entre

établissements : une comparaison européenne

\title{
Les chefs d'établissement face aux enseignants : enjeux et conflits de l'autonomie pédagogique
}

School principals faced with the teachers: stakes and conflicts of teacher autonomy

Los jefes de establecimiento frente a los docentes : lo que está en juego y

conflictos de la autonomía pedagógica

Schulleiter gegenüber Lehrer: Schwerpunkte und Konflikte in der pädagogischen

Autonomie

\section{Anne Barrère}

\section{QpenEdition}

\section{Journals}

Édition électronique

URL : http://journals.openedition.org/rfp/459

DOI : 10.4000/rfp.459

ISSN : 2105-2913

Éditeur

ENS Éditions

Édition imprimée

Date de publication : 1 juillet 2006

Pagination : 89-99

ISBN : 978-2-7342-1060-3

ISSN : 0556-7807

Référence électronique

Anne Barrère, « Les chefs d'établissement face aux enseignants : enjeux et conflits de l'autonomie pédagogique », Revue française de pédagogie [En ligne], 156 | juillet-septembre 2006, mis en ligne le 27 septembre 2010, consulté le 10 décembre 2020. URL : http://journals.openedition.org/rfp/459; DOI : https://doi.org/10.4000/rfp.459 


\section{Les chefs d'établissement face aux enseignants : enjeux et conflits de l'autonomie pédagogique}

Anne Barrère

L'article se propose d'envisager les relations entre chefs d'établissement et enseignants dans les collèges et lycées autour des nouveaux enjeux conférés par l'attribution d'un rôle pédagogique aux premiers, sans que n'ait été remise en question l'autonomie des seconds. A partir d'une enquête qualitative portant sur le travail quotidien des principaux et proviseurs, on analysera comment différentes tensions s'organisent autour de deux mouvements inverses. L'établissement s'approche de la classe au travers d'un certain nombre de dispositifs pédagogiques pris en charge et parfois animés par les directions d'établissement ; la classe sort d'elle-même, principalement à l'occasion de problèmes de gestion de classe auxquels les enseignants ne peuvent ou ne veulent faire face de manière isolée. On sera conduit enfin à définir l'établissement scolaire comme un espace de conflits en évolution, où au-delà des problématiques syndicales, l'expertise locale et le soutien aux enseignants sont amenés à jouer un rôle majeur.

Descripteurs (TEE) : conduite de la classe, chef d'établissement, enseignant du second degré, gestion d'établissement scolaire, style pédagogique.

'établissement scolaire est le cadre de vie et de travail quotidien de tous les acteurs de l'école. Mais son appréhension s'est transformée depuis les années 1980, et l'introduction de politiques publiques décentralisatrices et modernisatrices qui font de l'échelon local un cadre désormais important. Si tous les professionnels de l'éducation sont familiers et censés être acteurs de cette nouvelle donne, les chefs d'établissement la portent tout particulièrement, étant donné qu'elle a redéfini leur situation professionnelle, la revalorisation de leur statut allant de pair avec l'élargissement de leur mission (Blanchet, 1999 ; Pelage, 2000 ; Dutercq, 2003). En effet, face à une conception administrative et gestionnaire de la direction, s'impose l'idée d'une articulation étroite de l'organisationnel et du pédagogique, en particulier au 
travers de l'animation d'équipes et de projets, mais aussi en s'appuyant sur la diffusion d'une culture de l'évaluation centrée sur une obligation de résultats (Demailly, 2005). La dualité structurelle de l'organisation scolaire, bureaucratie professionnelle dont la cellule de base est la classe, où l'enseignant jouit d'une autonomie totale, mais reste aussi le maillon " exécutant " d'un certain nombre de prescriptions, se voit alors quelque peu brouillée (Tardif \& Lessard, 1999 ; Gather Thurler, 2000).

Mais cette redéfinition ne va pas sans un certain lot d'imprécisions ni même de paradoxes. Le point d'appui et la marge de manœuvre réelle de l'action pédagogique du chef d'établissement apparaissent incertains au moment même, d'ailleurs, où l'effort de la hiérarchie de l'Éducation nationale va dans le sens de l'instauration d'une coupure forte entre enseignants de base et personnels d'encadrement (Pelage, 2003). Le discours du nouveau management public prévaut alors sur celui de la pédagogie ; la question de la coordination et de la visibilité de l'action sont au centre des préoccupations des chefs d'établissement (Derouet, 2000). D'un autre côté, les enseignants ont évidemment un rôle à jouer dans cette redéfinition qui les concerne au premier chef, même si l'établissement n'est pas forcément un échelon qu'ils jugent pertinent de leur identité professionnelle (Demailly, 1998). C'est une part de l'autonomie au travail des enseignants qui se joue dans l'évolution de l'articulation entre l'encadrement des établissements et l'activité quotidienne des classes.

C'est à partir d'une étude qualitative du travail des chefs d'établissement que nous voudrions réexaminer cette situation (1). II s'agira moins d'analyser l'évolution de la professionnalité que suppose cette entrée en pédagogie que de la resituer dans l'ensemble des tâches qu'ils effectuent quotidiennement dans les établissements. Très concrètement, leur action pédagogique s'incarne dans la mise en place d'un certain nombre de dispositifs prévus par l'institution et rendus possibles par les marges de manœuvre de la gestion de moyens depuis la décentralisation. Mais, parallèlement, un certain nombre de problèmes de la relation pédagogique sont gérés dans l'établissement, essentiellement au travers des problèmes d'indiscipline et de gestion de classe. L'analyse de ce double mouvement permet de rendre compte de la manière dont le travail des chefs d'établissement et des enseignants s'articule aujourd'hui autour d'un nouvel enjeu pédagogique, que l'ancienne définition des missions des directions ne laissait que très peu exister. II mène aussi à tenter de construire l'établissement scolaire comme un espace de conflits en transformation.

\section{QUAND L'ÉTABLISSEMENT ENTOURE LA CLASSE : L'ÉMERGENCE D'UNE PÉDAGOGIE D'ÉTABLISSEMENT}

Dans les discours sur leurs missions, les chefs d'établissement cherchent à concilier la modernisation organisationnelle, parlant massivement de leur désir de "faire bouger, faire avancer les choses ", davantage en tout cas que dans leur ancien métier (2), et l'idée de progrès social par l'école, largement comprise à partir de l'égalité des chances et de la centration sur l'élève présente dans la loi d'orientation de 1989. Mais "faire bouger les choses ", c'est aussi, bien sûr, faire bouger les pratiques enseignantes, dont dépendent, ils le savent bien, une grande partie de leur action et de leurs résultats dans l'établissement.

\section{Observateurs critiques, acteurs prudents}

Or, les chefs d'établissement savent aussi que les enseignants sont loin d'approuver tous l'élargissement de leurs fonctions à une sphère proprement pédagogique (Guillaume, 1997). Les entretiens sont émaillés d'expressions prudentes: ils parlent "de ne pas brusquer», « de ne pas braquer» les enseignants. L'extension qu'ils donnent à leur action pédagogique en est d'ailleurs révélatrice. En effet, hormis une minorité de chefs d'établissements ayant eu, en tant qu'enseignants ou éducateurs, un passé d'innovateurs (3), elle est davantage définie par eux en termes d'organisation (emploi du temps, constitution des classes et des équipes enseignantes) ou d'animation (mise en place de réunions, impulsion de projets et d'actions) que de réflexions sur les méthodes et les pratiques, entre théorie et expérience (Fabre, 2006). Alors que leur vécu d'enseignant s'éloigne dans le temps, ils n'ont pas de convictions ni de connaissances forcément très précises en la matière. S'ils regrettent à l'unanimité de ne pas avoir assez de temps pour ce qu'il est convenu d'appeler le pilotage pédagogique de l'établissement (Dupriez, 2002), ce terme désigne encore un autre domaine, les tâches de traitement sur les indicateurs de résultats et des performances de l'établissement, à l'heure où il leur est de plus en plus demandé d'être des experts locaux de leur propre établissement scolaire.

Pourtant, on ne peut pas dire en un sens qu'ils manquent de convictions pédagogiques, si ce n'est qu'elles sont surtout basées sur une sorte de " théologie négative ", instrumentée par la critique quotidienne des pratiques enseignantes. Le thème le plus important tourne autour de l'évaluation des élèves, 
dont ils prennent connaissance lors des conseils de classe, ou de la comparaison des contrôles continus et des évaluations plus standardisées que constituent les examens. Ils craignent bien davantage les enseignants trop sévères et démotivants que ceux qui seraient "laxistes ", l'évaluation étant créditée d'un pouvoir d'humiliation (Merle, 2005) pouvant susciter des comportements de refus. Si beaucoup sont d'avis de "sortir du frontal ", c'est qu'il décourage certains élèves. Ce sont surtout la motivation et ses blocages qui sont au centre des préoccupations pédagogiques des chefs d'établissement. En positif, les chefs d'établissement se rejoignent dans un appel général et intense à la collaboration et au travail en équipes enseignant, et du dépassement d'une culture professionnelle jugée globalement individualiste, un appel dont la plupart estime d'ailleurs qu'il est bien reçu par une grosse minorité des enseignants en poste. L'action dans l'établissement, sous forme de projets, a tendance à devenir une norme de jugement professionnel : le bon enseignant est désormais celui qui ne " fait pas que ses heures " et qui manifeste par son dynamisme et sa participation sa préoccupation de la bonne marche de l'établissement, en plus de celle de sa classe.

\section{Des dispositifs pédagogiques, à mi-chemin entre classe et établissement}

Un certain nombre de dispositifs donne actuellement aux chefs d'établissement l'occasion d'incarner cette critique engagée. S'ils n'ont pas tous la même origine ni finalité, ni la même ampleur (4), ils nécessitent de la part des directions d'établissement à la fois un travail d'organisation pédagogique obligatoire, puisqu'ils font l'objet de circulaires ministérielles et un travail relationnel plus ou moins poussé d'animation au quotidien (5). Malgré leur diversité, ils ont en commun plusieurs caractéristiques. D'une part, ils ne concernent pas tous les enseignants, et il faut donc trouver suffisamment de volontaires, et parfois convaincre les enseignants réticents pour les animer. Ensuite, ils ne concernent pas des groupes-classes constitués, ordinaires, mais des fractions d'une même classe, ou des regroupements de différentes classes. Ils sont donc en principe des occasions de pédagogies différenciées ou individualisées. Enfin, ils sont un vecteur fort du travail en équipe, dans la mesure où ils s'accompagnent en général de la mise en place de dispositifs de coordination, de mise en commun ou d'évaluation (Braxmeyer \& Do, 2002). Pour cette raison, ces dispositifs constituent en quelque sorte aujourd'hui, un espace pédagogique intermédiaire entre classe et établissement, l'expression de pédagogie d'établissement, que nous emploierons pour les désigner ensemble ne signifiant pas, précisément, qu'ils soient unifiés ou discutés en tant que tels.

Cet espace est constitué de quatre grands types de dispositifs, qu'il faut décrire succinctement. Tout d'abord, de tous les dispositifs de remédiation et d'aide individualisée. L'idée d'aide ciblée aux élèves en difficulté dans certaines matières, proposée sous le ministère Jospin en 1988, a surtout été mise en œuvre partir de 1997 au collège et de 1999 au lycée. Se généralise ainsi une pédagogie dite de soutien, la plupart du temps sous forme de «modules " ou de groupes d'aide ou de besoin, en majorité animés par des enseignants volontaires, mais parfois aussi par des aide-éducateurs, en particulier lorsque les enseignants y sont un peu réfractaires. Au collège Salvador Allende, les élèves de sixième sont pris en charge deux heures et deux jours par semaine pour des modules d'aide, d'abord portant sur des « méthodologies transversales » comme la tenue du cahier de textes, l'apprentissage d'une leçon, puis sur des acquis disciplinaires plus précis en français et en mathématiques (6). Cette pédagogie du soutien peut être vue comme une forme édulcorée de pédagogie différenciée (Houssaye, 1999) et critiquée comme telle, mais elle désormais largement passée dans les habitudes scolaires, en dépit du scepticisme des chercheurs sur leur efficacité (Duru-Bellat, 2002).

En second lieu, des dispositifs interdisciplinaires ont été introduits sur la même période: Itinéraires de découverte au collège (7), Travaux personnels encadrés au lycée général et technologique (8), Projets professionnels à caractère disciplinaire au lycée professionnel. Ils sont centrés sur la mise en relation possible des disciplines d'enseignement autour d'une problématique, le recours à des supports d'apprentissage variés, et surtout sur la mise en œuvre d'une démarche plus autonome et réflexive de la part des élèves (Kerlan, 2003 ; Boulet, Delattre \& Sagot, 2003). Ils font l'objet de bilans plus ou moins optimistes de la part des chefs d'établissement.

Le troisième type de dispositifs est d'une certaine manière le plus ancien, puisqu'il est constitué par les actions du projet d'établissement, obligatoire à partir de 1989, mais il se décline différemment selon les contextes. Ce n'est que pour une minorité de chefs d'établissement interrogés, sept seulement pourraiton dire, au vu de l'importance du thème dans la littérature officielle, qu'il constitue un ensemble cohérent au sens fort du terme, "plus que la somme des actions » (9). Le cas de Salvador Allende est en la 
matière particulièrement emblématique puisque, en dépit de multiples actions très diverses et réussies (venue d'un romancier et d'une résistante, actions sur la circulation routière, projet sur « les valeurs " primé nationalement, actions en partenariats avec un dessinateur de bande dessinée), l'équipe de direction trouve que le collège manque d'un "projet fédérateur » qui permettrait de coordonner davantage les différentes actions.

Dans bien d'autres établissements, l'idée d'une amélioration des résultats, ou de l'ambiance de l'établissement ne s'appuie pas forcément de manière très nette sur le projet d'établissement, même si les sorties ou les actions diverses sont en général vues comme des moyens de valoriser les élèves et d'améliorer les relations. II faut rappeler aussi que dans certains établissements, le projet d'établissement est bien davantage animé par les acteurs de la « vie scolaire » : conseillers principaux d'éducation, conseillers d'orientation ou aide-éducateurs que par les enseignants eux-mêmes.

Enfin, le quatrième type de dispositifs ne concerne que certains établissements, classés en REP ou "zone violence", et il est censé avant tout faire face à des problèmes d'incivilités ou de décrochage. Classes-relais, dispositifs individualisés d'aide à l'insertion, école ouverte, sont autant ou même davantage centrés sur les problèmes de socialisation que sur l'apprentissage. A Salvador Allende, un dispositif de tutorat, animé par une enseignante de mathématique et regroupant une dizaine d'enseignants et le médecin scolaire a pour vocation d'anticiper les problèmes des élèves difficiles en les repérant dès le début de l'année et en leur attribuant un tuteur. Par contre, les enseignants de ce collège refusent depuis plusieurs années de participer aux dispositifs d'École ouverte, consistant à organiser des ateliers divers, miludiques, mi-scolaires dans les établissements durant les vacances scolaires.

Si l'ensemble de ces dispositifs contribue à l'émergence d'une pédagogie d'établissement, il ne remet pas ouvertement en question le principe de l'autonomie pédagogique des enseignants dans leur classe. Pourtant, il l'interroge tout de même en donnant un contenu au travail en équipe, en invitant à des formes d'individualisation pédagogique, à dépasser certains cloisonnements curriculaires, à introduire des pratiques d'évaluation différentes. Parfois porteurs de rupture dans l'ordinaire de la classe (Bouveau \& Rochex, 1997), parfois censés précisément l'améliorer en permettant à des élèves de mieux y réussir, ils sont en tout cas les supports privilégiés de l'action pédagogique des directions d'établissement.

\section{QUAND LA CLASSE S'OUVRE SUR L'ÉTABLISSEMENT : VERS UNE GESTION COLLECTIVE DES TÂCHES DE L'ORDRE SCOLAIRE ?}

Si pour un discours expert, les problèmes de gestion de classe sont solubles dans les compétences pédagogiques, un "bon " cours, adapté à tous et à chacun ne pouvant que bien se passer, force est de constater à partir des enquêtes empiriques qu'il s'agit là d'une représentation idéale qui correspond peu à la réalité. Le travail enseignant peut être analysé au travers de l'autonomisation relative d'une sphère relationnelle qui déborde, tout en la conditionnant, la sphère pédagogique proprement dite ou qui en constitue un préalable encombrant, un "sale boulot " consistant à gérer les interactions soumises à bien des aléas et à l'humeur des groupes. Les problèmes d'ordre scolaire, normalement gérés en huisclos, sortent alors de la classe par le biais des difficultés professionnelles des enseignants, sans qu'ils soient l'objet de prescriptions explicites et d'ailleurs, en général, en dehors d'une volonté claire des enseignants.

Aucun chef d'établissement interrogé ne considère comme satisfaisants les moyens traditionnels à disposition lors du constat de problèmes récurrents de discipline. L'appel aux corps d'inspection, seuls responsables de l'évaluation pédagogique, ou la baisse de la note administrative (10) sont décrits comme assez peu efficaces et de plus, parfois sources de conflit ou de dévalorisation supplémentaire. Bien des chefs d'établissement peuvent être alors tentés de ne rien faire, préférant le prudent et bureaucratique " je ne veux pas le savoir". Mais ce n'est pas toujours possible, puisque dans les cas les plus graves, la sécurité des personnes, dont ils sont responsables juridiquement, peut être engagée. Pour bien d'entre eux, ce n'est pas non plus souhaitable, le climat d'établissement (11) mis en péril par trop de classes agitées étant une de leurs préoccupations centrales. En fait, l'ordre scolaire est de facto un problème dont l'espace réel est l'établissement, même s'il n'est pas toujours reconnu comme tel. II est l'objet d'une division du travail d'autant plus problématique que les problèmes y sont intenses (Payet, 1997 ; van Zanten, 2001 ; Kherroubi, 2003). On peut alors distinguer, à partir des tâches effectuées par les chefs d'établissement, trois grandes manières de les prendre en compte.

Au quotidien, et même s'ils ne sont pas en première ligne face aux élèves difficiles, qui sont en général 
d'abord vus par les surveillants ou la vie scolaire (Masson, 1999 ; Andréo, 2005), ils sont tout de même amenés à gérer un certain nombre d'incidents qui se passent à l'intérieur des classes, soit au travers des exclusions de cours, soit au travers d'actions de médiation entre élèves et enseignants, ou entre enseignants et parents d'élèves. À Salvador Allende, le conflit entre un enseignant et une élève s'étendra à la famille lors d'une réunion de parents d'élèves et finira par un changement de classe de l'élève, l'agressivité ayant atteint un seuil tel que l'enseignant paraît ne plus pouvoir supporter la présence de la jeune fille. II accuse d'ailleurs l'équipe de direction de trop l'écouter, et de ne pas assez le soutenir. De manière plus préventive, des coups de main ponctuels sont apportés à certains enseignants. Ce proviseur de lycée professionnel a patrouillé durant des mois à heures fixes devant la porte, laissée ouverte, d'un enseignant mis en difficulté par une classe. Quelques chefs d'établissement viennent parfois résoudre les problèmes dans les classes, tout en sachant que le " coup de gueule" et les « remontées de bretelles » ont leurs limites, et surtout leur paradoxe, puisque c'est surtout sa propre autorité que l'on renforce en tentant d'étayer celle de l'enseignant.

D'une manière moins ponctuelle, un certain nombre de chefs d'établissement disent s'engager dans des interactions de conseils et de soutiens en matière de gestion de classe, une des caractéristiques des personnels de direction non enseignants antérieurement étant qu'ils disent s'y aventurer moins, par peur de se voir refuser une légitimité en la matière. Ces entretiens ressemblent parfois à des entretiens d'évaluation ou de progrès, tels qu'ils existent dans les entreprises, sans que les chefs d'établissement ne reconnaissent forcément explicitement cette dimension. Le problème est alors l'aspect consommateur de temps et d'énergie de ce travail d'écoute, qui doit parfois se répéter. À Salvador Allende, une enseignante en difficulté sera le centre des préoccupations toute l'année d'observation, sans que ses problèmes de gestion de classe ne s'améliorent durablement. Le chef d'établissement interviendra dans sa classe, le groupe d'élèves le plus difficile devra rédiger une petite lettre d'excuse dans le bureau de la principale adjointe, après un cours particulièrement agité. Pourtant, le soutien de l'équipe de direction lui permet de gérer la situation, et même de connaître un mieuxêtre personnel, semble-t-il, vers la fin de la période d'enquête.

Enfin, la gestion de classe peut être prise en charge au niveau de l'organisation pédagogique elle-même.
La répartition des élèves, l'attribution des classes et les emplois du temps des enseignants les plus en difficulté sont évidemment l'occasion d'arbitrages et de dilemmes pour les équipes de direction, toujours conscientes qu'elles organisent aussi d'une certaine façon, en partie à l'aveugle, en partie par des stratégies conscientes de regroupement des élèves ou de construction d'équipes pédagogiques, les problèmes futurs de gestion de classe. Dans un gros lycée polyvalent, un proviseur essaie d'objectiver autant que possible les "risques " d'incidents en les estimant classe par classe, à la pré-rentrée, et en les formalisant sur des fiches. Mais il s'agit là d'un cas isolé. En fait, les équipes ne gèrent pas pour le moment explicitement et surtout collectivement l'organisation de l'établissement en mettant en son centre les problèmes de gestion de classe. Beaucoup laissent agir le cercle vicieux de l'enseignant en difficulté confronté à des classes faibles ou difficiles, parce que la matière y est dotée d'un moins fort coefficient à l'examen ou par désir d'épargner des classes scolairement plus prometteuses. Les problèmes de gestion de classe, restent, il est vrai, trop difficiles à évoquer dans les collectifs enseignants, tant ils sont porteurs de remise en question indissociablement professionnelle et personnelle (Barrère, 2002). Pourtant la gestion des difficultés enseignantes au quotidien est évoquée par tous les chefs d'établissements sans exception, y compris dans des établissements à publics favorisés ou de centre ville (12).

Si les deux mouvements interrogent à la fois l'autonomie du travail enseignant, et les tâches d'encadrement, ce n'est pas de la même manière. Le premier entend par pédagogie l'instauration de cadres de travail qui ont en commun une certaine différenciation, et leur caractère innovateur ; le deuxième concerne la difficulté à gérer la forme scolaire ellemême, au sens de la cohabitation dans un espace et pour un temps donné d'un enseignant et d'un groupe d'élèves. Le premier correspond, pour les équipes de direction, à un ensemble de tâches prescrites alors que le deuxième correspond à des tâches qui s'imposent à elles dans le quotidien de certains établissements. On peut penser que les enseignants dans leur ensemble pourraient voir d'un œil plus positif le deuxième processus, susceptible de leur apporter aide et soutien dans une tâche dont ils sont responsabilisés à l'extrême, que le premier, plus intrusif dans leur sphère d'expertise, mais le tabou qui pèse sur les problèmes d'autorité ordinaire et la manière dont se présente aujourd'hui la pédagogie d'établissement interdisent précisément un contrepoint aussi simple. 


\section{UNE CONFLICTUALISATION PAR DÉFAUT ?}

L'établissement scolaire est un lieu alternativement de débats et de moments plus silencieux de suspension des conflits possibles sur les situations vécues ou les actions à mener. Les chefs d'établissements, comme tous les cadres ou responsables, sont amenés à " agir dans plusieurs mondes ", en articulant les références à une pluralité de références possibles de l'action éducative (Derouet \& Dutercq 1998 ; Derouet, 1999), d'une manière qui ne correspond pas forcément aux arbitrages du monde enseignant. Le «management éducatif » qui tente de concilier la référence au civisme et à l'efficacité, voire aux préoccupations de renom, dans un monde de concurrence scolaire larvaire, est vu avec méfiance par certains enseignants, accepté par d'autres, sans que d'ailleurs les débats ne s'expriment forcément ouvertement. Mais ce qui nous intéresse ici, c'est la manière dont les transformations évoquées, arrivent - ou non - à devenir des enjeux explicites dans l'établissement scolaire.

\section{La difficile conflictualisation de la pédagogie d'établissement et son déplacement sur le problème de l'autorité}

La pédagogie d'établissement est difficile à discuter pour plusieurs raisons. D'une part, si le processus de professionnalisation visant à faire des enseignants des experts de la pédagogie rend compte d'un certain nombre d'évolutions, et en particulier de la constitution des IUFM, il ne se traduit pas forcément, dans le quotidien des établissements, par une augmentation de la capacité à en débattre. Pourtant, ces dispositifs pourraient être mis à l'épreuve de certaines critiques expertes si elles étaient relayées et portées au local, en particulier celles qui instruisent la déliaison de la modernisation pédagogique et de la démocratisation, soupçonnant la pédagogie de projet de contribuer à dévaloriser encore davantage le travail scolaire ordinaire ou l'effort d'abstraction nécessaire aux apprentissages par des pédagogies abusivement contextualisées (Bouveau \& Rochex, 1997 ; Terrail, 2002 ; Kherroubi \& Rochex, 2004). Mais la difficile circulation entre connaissances expertes et praticiens (Rayou, 2002) ainsi que l'appui de ces dispositifs sur des constats d'évidence comme la nécessité de soutenir ou de remotiver les élèves en difficulté ne favorisent guère les interrogations sur ces dispositifs (13).

Par ailleurs, pour une partie des enseignants, ils s'inscrivent en relative continuité avec leurs pratiques professionnelles en classe : tentatives d'indivi- dualisation, travail sur la motivation sont aussi des tâches quotidiennes pour eux. Surtout, les groupes de remédiation, ou de travail sur projets, et a fortiori de prises en charge des élèves difficiles permettent aussi en général des relations plus paisibles, et atténuent les difficultés de la gestion de classe. Ainsi, audelà parfois de toute considération pédagogique, ils permettent aux enseignants d'avoir affaire à un plus petit nombre d'élèves, satisfaisant ainsi une de leurs revendications les plus stables (14).

La plupart des chefs d'établissement interrogés apparaissent d'ailleurs à la recherche d'une hiérarchie pédagogique intermédiaire dans l'établissement (15), et cherchent à la constituer en s'appuyant sur les professeurs principaux, les coordonnateurs disciplinaires ou les porteurs de divers dispositifs. Mais elle émerge à peine, et les chefs d'établissement la considèrent dans l'ensemble pour l'instant davantage comme une structure d'appui et d'aide à leur propre travail, voire comme un moyen de contourner les syndicats. Un proviseur de lycée, mais c'est un cas extrême, parle même d'enseignants qui lui seraient «inféodés». Du coup, le travail d'expertise quotidienne est très souvent effectué par les équipes de direction ellesmêmes, et sans vraiment de débat, lorsque, par exemple, elles jugent trop important le volume des sorties et des voyages au regard de projets plus scolaires, ou qu'elles contestent des projets au vu de leur manque d'efficacité supposé en termes de résultats.

Les recherches ont souvent classé les chefs d'établissement selon leur degré d'interventionnisme pédagogique (Guillaume, 1997 ; Cousin, 1998). Si les chefs d'établissement les plus volontaristes ont été crédités d'une efficacité supérieure par certaines recherches (Ballion, 1986), force est de constater, au vu de notre propre enquête, qu'un certain nombre d'entre eux font face à des conflits très vifs avec les enseignants. Mais, de manière frappante, ces conflits ne concernent pas la pédagogie d'établissement qu'ils tentent de mettre en place mais en général leur " autoritarisme", leurs styles de relations avec les enseignants. Dans un lycée polyvalent, la restructuration d'un établissement autour du développement durable, articulant un projet d'établissement extrêmement ambitieux à des ouvertures de section n'est pas mis en question par les enseignants. La restructuration d'un autre lycée en divers services, consacrés aux nouvelles technologies, à la gestion des ressources humaines ou à la pédagogie n'est pas non plus critiquée frontalement, et le projet d'établissement est voté facilement au conseil d'administration. Par contre les deux proviseurs font face à des conflits très 
durs, en particulier avec les représentants du SNES, qui les accusent d'abus de pouvoir, et même pour l'un d'entre eux, en conflit avec trois enseignantes, d'être un «tyran misogyne ».

\section{La conflictualisation en creux de la gestion de classe par le problème des sanctions}

La construction des problèmes de gestion de classe en enjeu collectif n'est pas plus facile, bien au contraire. En effet, les aléas du désordre scolaire restent vécus comme la responsabilité individuelle de l'enseignant, alors même qu'ils résultent de transformations macro-sociales, où la massification de l'enseignement et les évolutions des rapports d'autorité entre générations sont au premier plan. Mais peu socialisés, ils restent facilement l'objet de culpabilité ou de honte et constituent un indice synthétique d'échec professionnel (Barrère, 2002a). La gestion de classe apparaît comme le chaînon manquant d'un travail en équipes qui pourtant pourrait y trouver une véritable justification, en permettant de transférer au collectif une partie de la charge mentale que font peser ces problèmes sur l'exercice du métier (Barrère, 2002b).

Mais il n'en reste pas moins que les enseignants ressentent et expriment le besoin d'être soutenus en dehors de la classe par les personnels de la vie scolaire ou par les directions. C'est alors par le biais de l'absence de soutien que les enjeux de la gestion de classe sont conflictualisés, pour ainsi dire en creux, de deux manières différentes. Tout d'abord au travers des sanctions. Les chefs d'établissement interrogés disent tous ressentir une pression globale à la sanction de la part des enseignants, qui sont prompts à les taxer de laxisme ou de faiblesse. Pourtant, il leur arrive très fréquemment d'interroger une demande de sanction qu'ils jugent trop lourde ou d'essayer de ralentir la réactivité enseignante en cas d'incidents en interprétant au cas par cas les incivilités des élèves en fonction des contextes. Soutenir systématiquement les enseignants par les sanctions est tout sauf évident pour eux car ils développent dans l'ensemble un discours critique sur l'« incivilité enseignante » qui les amène à considérer que, dans un certain nombre de cas, les torts sont plus que partagés. Enseignants injurieux, coutumiers de la «brimade orale», ou n'hésitant pas devant « les noms d'oiseaux ", comportements à la limite du racisme, ou même acharnement obsessionnel sur tel ou tel élève, injustices flagrantes, les anecdotes sont légion dans les entretiens. Mais les conflits ponctuels en conseils de discipline ou en conseils de classe ne sont que rarement l'occasion d'une réflexion collective. Et de toutes façons, elles ne sont qu'une manière bien particulière et partielle d'aborder les problèmes de gestion de classe, sans remise en question ou explicitation des pratiques enseignantes.

La deuxième manière de conflictualiser les demandes de soutien, réelles ou symboliques, se focalise alors sur la question de la présence physique du chef d'établissement. Souvent retenus à l'extérieur pour de multiples activités institutionnelles, partenariales ou de représentation (16), ou trop enfermés dans leur bureau, dans une définition bureaucratique du métier, certains chefs d'établissement reconnaissent d'ailleurs eux-mêmes qu'ils s'exposent à ce reproche. Comment un chef d'établissement absent peutil alors soutenir les enseignants et effectuer ce travail relationnel si précieux lorsque la classe déborde d'elle-même?

\section{La traduction syndicale des conflits de l'autonomie enseignante}

Si bien des enseignants appellent «patron » le chef d'établissement, avec d'ailleurs, une dose de distance humoristique variable, cette appellation prend plus ou moins de consistance en fonction des rapports réels entretenus par les équipes de direction avec les sections syndicales, principalement celles du SNES, le syndicat majoritaire (17). Les chefs d'établissement se classent spontanément entre ceux qui ont - ou n'ont pas - « des CA durs ", les conseils d'administration étant les lieux d'expression institutionnalisés des conflits avec les syndicats.

Pourtant la traduction syndicale des enjeux de l'autonomie enseignante témoigne là aussi de la difficulté actuelle à les considérer en tant que tels.

Sur le plan pédagogique, les syndicalistes n'ont pas de positions strictement différentes de l'ensemble de leurs collègue (Robert \& Mornettas, 1994) et s'ils sont parfois aussi des innovateurs pédagogiques dans leurs classes, ce n'est pas vraiment sur ce terrain-là qu'ils conflictualisent les situations. Lorsqu'ils le font, c'est donc largement en prenant acte du déplacement vers les relations d'autorité et du thème des abus de pouvoir. Le proviseur du lycée à projet environnemental, déjà évoqué, se voit contester au conseil d'administration à propos des attributions de cette même instance, les enseignants désirant y voir traiter des sujets comme les dates de conseil de classe, qui lui paraissent triviaux. L'année de l'enquête, deux autres 
conflits éclatent au CA : I'un à propos de la date de libération des élèves de terminale, qu'il veut repousser pour leur permettre de réviser au lycée, l'autre à propos de la suppression d'une plage d'évaluation commune. Dans l'autre lycée évoqué, c'est au contraire l'établissement de plages d'évaluations communes qui sera contesté et non le projet global de restructuration du lycée. Ce sont les modifications de l'offre scolaire par l'ouverture d'options, ou les dispositifs de lutte contre le décrochage et la violence qui donnent lieu sans doute aux débats les plus institutionnalisés dans les établissements (18). L'ouverture de sections européennes dans un lycée, nécessaire selon le proviseur pour affronter la concurrence locale, sera très mal acceptée par une partie des enseignants. Si elle sera effective, le conflit participera à une situation d'affrontement qui conduira au départ du chef d'établissement. Dans un collège, un dispositif-relais interne sera l'objet d'un grave conflit, les débats portant moins sur sa nature que sur la décision autoritaire de le supprimer.

En fait, une grande partie des conflits syndicaux tels qu'ils s'expriment au conseil d'administration concerne davantage les tâches plus traditionnelles de gestion administrative ou financière de l'établissement, le vote du budget étant parfois l'objet d'âpres discussions sur l'utilisation des fonds (19). Les syndicats ont, selon les chefs d'établissement, une propension trop systématique à l'épargne, ce qui peut masquer des conflits sur l'utilisation des moyens. En dehors du CA, l'évaluation formelle des enseignants peut être également un motif fréquent de conflits, lorsque les chefs d'établissement utilisent leurs marges de manœuvre à la baisse, en refusant d'avancer une note en stricte fonction de l'ancienneté, mais aussi de soutenir ou d'accréditer des pratiques pédagogiques qui leur paraissent injustifiables. Les différends de l'évaluation s'articulent certes avec les nouveaux enjeux - ce sont parfois des enseignants insuffisamment participatifs au niveau de l'établissement ou ayant de graves problèmes de gestion de classe qui sont concernés, mais ils sont rarement posés comme tels. Dans les pires des cas, les conflits syndicaux dépassent des enjeux circonscrits : ils sont alors généralisés à l'ensemble de l'établissement, éventuellement en divisant les équipes enseignantes. L'établissement devient alors un champ de bataille, les conflits produisent un climat général de tension qui rend les désaccords globaux, sans plus de localisation possible, et susceptibles de dérapages dans le monde très humain des agressions personnelles (Derouet \& Dutercq, 1998), dont les chefs d'établissement reconnaissent qu'ils peuvent alors envahir leur vie privée (20). Ainsi, dans l'ensemble, la conflictualisation syndicale dans l'établissement se fait bien davantage en entérinant le déplacement des enjeux pédagogiques sur les questions d'abus d'autorité et de déficit de présence qu'en les considérant en tant que tels, ainsi qu'en atteste d'ailleurs la place réduite des questions pédagogiques dans les conseils d'administration. Elle ne lève pas non plus les tabous sur les problèmes de gestion de classe ordinaire, à moins qu'ils ne soient durcis, dans les cas les plus extrêmes, en termes de violence scolaire, et puissent alors s'exprimer en termes de sécurité des personnels et de revendications de moyens.

\section{CONCLUSION}

Si le rôle pédagogique des chefs d'établissement entre bien en tension avec les contours de l'autonomie enseignante, si la pédagogie d'établissement et le soutien à la gestion de classe sont des enjeux quotidiens de la vie des collèges et lycées, ils sont aujourd'hui moins l'objet de débats formalisés que d'accords tacites. Certains chefs d'établissements échangent en effet une implication dans l'établissement contre un soutien potentiel, une reconnaissance des difficultés quotidiennes des équipes. L'échange est d'autant moins coûteux qu'une partie des enseignants au moins adhère aux nouveaux dispositifs pédagogiques ou au projet d'établissement; il est d'autant plus avantageux pour les enseignants qu'ils éprouvent au quotidien ce besoin de soutien. C'est pour cette raison qu'il est potentiellement bien davantage présent dans les établissements difficiles (van Zanten et al., 2002), pouvant être décrit alors comme un processus de mobilisation.

A contrario, les cas de figures les plus conflictuels se rencontrent dans des établissements où certains chefs d'établissements combinent un fort interventionnisme pédagogique et un déficit relatif de soutien, qu'il se manifeste par ce que les enseignants considèrent comme une faiblesse dans la prise de sanctions ou par un désengagement de l'établissement. Si ces conflits sont alors vécus par les directions d'établissement comme un face à face entre réforme organisationnelle et "résistance enseignante ", taxée d'individualisme structurel ou de corporatisme, soutenue par des syndicalistes qu'ils accusent parfois « de mauvaise foi ", on voit bien comment on peut les lire à partir des tensions actuelles de l'autonomie au travail. 
Ces enjeux sont peu conflictualisés pour l'instant, à un moment où les syndicats, dans le monde enseignant comme ailleurs, peinent à trouver leur place face à une expertise qui se fait parfois largement en dehors d'eux (Durand, 2004) et à des dispositifs d'écoute des travailleurs où ils sont parfois pris de vitesse dans les directions (Dejours, 1998). Or, débattre des dispositifs pédagogiques dans un contexte précis, de leur évaluation, discuter également les constats faits sur un établissement à partir des indicateurs qui en rendent compte, sont de réels enjeux, alors même que, malgré leur manque de temps, les directions d'établissement s'acquittent bien souvent seules de ce travail d'expertise ordinaire. C'est aussi en se reconstruisant de manière plus collective, autour des problèmes quotidiens du travail enseignant que les enjeux de l'autonomie deviendront ceux de l'établissement comme démocratie locale.

Anne Barrère anbarrere@nordnet.fr

Université Lille 3

Équipe de recherche sur les interactions professions, éducation, orientation (Proféor)

\section{NOTES}

(1) L'enquête a été effectuée de 2003 à 2005, à partir de quarante entretiens semi-directifs longs (deux heures environ) effectués dans des établissements de la région Nord-Pas-de-Calais (vingt en collèges dont cinq en Réseau d'éducation prioritaire (REP), dix lycées généraux et technique, dix lycées professionnels) et d'une observation ethnographique d'une année complète (2004) d'une équipe de direction dans un collège situé en REP du Nord-Pasde-Calais. Ce collège de 640 élèves est anonymé ici sous le nom de collège Salvador Allende. Le principal est en poste depuis quatre ans au moment de l'enquête et la principale adjointe depuis trois ans. Comme dans toute enquête qualitative de inductif, le raisonnement est construit à partir du matériel, qui peut aussi servir à l'illustrer, ce que nous ferons davantage en référence à l'observation qu'avec des citations d'entretiens, pour des raisons d'espace. Cependant, la partie sur les conflits fera appel à d'autres situations évoquées dans les entretiens, l'équipe de direction de Salvador Allende n'en vivant pas de majeurs. Ce collège dont les résultats au brevet sont inférieurs à la moyenne académique, est engagé dans un effort, couronné de succès, de baisse des incivilités et d'amélioration de sa réputation.

(2) Les chefs d'établissement restent encore très majoritairement d'anciens enseignants, même si leur origine a tendance à se diversifier en particulier à la faveur de l'extinction du corps des PEGC qui représentait $30 \%$ des chefs d'établissement dans les années 1970 et d'une montée de personnels issus du premier degré et de l'enseignement adapté. En dehors de l'enseignement, la proportion de personnels issus des métiers de l'éducation et de l'orientation augmente également. Pour le concours $2004 \mathrm{de}$ la deuxième catégorie, par exemple, les reçus étaient à $50 \%$ des certifiés, à $13,4 \%$ des enseignants de lycée professionnel, à $11,5 \%$ des enseignants du premier degré. Les personnels d'éducation et issus de l'enseignement adapté représentaient $21 \%$ des candidats et les personnels d'orientation $3 \%$. Le concours de première catégorie n'est présenté, par définition que par des agrégés, la réforme du statut des personnels de direction mise en œuvre à partir de 2001 assouplissant les voies de passage entre les deux catégories (France, 2004).

(3) Dans l'enquête, six chefs d'établissement appartiennent à ce groupe et cherchent nettement à promouvoir des pratiques de pédagogies différenciées ou de projet.

(4) En Réseau d'éducation prioritaire ou en «zone sensible » et "zone violence", des moyens supplémentaires donnent évidemment plus de marge de manœuvre à ce niveau aux directions d'établissement comme le font remarquer surtout... les chefs d'établissement des collèges dits " favorisés ".

(5) D'autres résultent, de plus en plus, de négociations, voire d'initiatives des collectives locales, qui sont amenés à développer depuis quelques années de véritables politiques éducatives plus ou moins en tensions avec les politiques du ministère. Les dispositifs peuvent alors parfois s'additionner dans un même établissement (Dutercq, 2000).
(6) II est à noter que dans plusieurs collèges REP où nous avons enquêté, les dispositifs de soutien servent aussi à aider les meilleurs élèves, dans la perspective d'une orientation en lycée, et de crainte que le niveau moyen du collège ne les pénalise. Certaines décisions de constitution ou de composition de classes relèvent aussi de cette sphère d'action, lorsque par exemple sont constituées en lycée des classes de doublants ou "à projets ", qui contreviennent au principe de l'hétérogénéité, mais sont censées s'attaquer de manière plus spécifique aux problèmes d'échec scolaire.

(7) Appelés parcours diversifiés dans leur première formulation en 1996, puis travaux croisés en 1998, ils s'appellent Itinéraires de découverte depuis 2002

(8) Expérimentés en 1999, ils sont généralisés en 2000, supprimés en Terminale par la réforme Fillon, en 2004

(9) Plusieurs rapports officiels sont cependant tout à fait lucides, sur les faiblesses effectives du pilotage par le projet (voir par exemple, Dulot, 1998)

(10) Rappelons qu'elle comprend un item appelé « autorité, rayonnement ".

(11) La notion de climat d'établissement, restée floue en tant que notion heuristique est cependant très fédératrice de bien des évaluations qualitatives des établissements de la part de leurs acteurs, qui raisonnent très souvent en ces termes. Pour une mise en perspective de la notion dans les travaux de base ethnographique, voir Derouet, 1987.

(12) De manière troublante, le nombre d'enseignants en difficulté chronique font l'objet de la même estimation, quel que soit l'établissement, sa taille, et la difficulté de son public : de deux à cinq, c'est-à-dire une infime minorité. Ce chiffre témoigne sans doute de l'adaptation de la définition de "l'enseignant à problèmes " au contexte de l'établissement.

(13) A contrario, en marge de l'enquête, l'organisation d'une journée de formation des professeurs principaux de lycée dans l'Est de la France, sur le thème de l'évaluation des élèves, où j'intervenais à partir d'un exposé de recherches antérieures, fut l'occasion d'une réflexion contradictoire sur la proposition de création d'une seconde de remédiation pour des élèves faibles. J'y développais des résultats de recherche montrant les dangers des regroupements par niveau (en m'appuyant sur les travaux de l'IREDU en particulier). Une année après, rencontrant par hasard une des enseignantes du stage, j'apprendrai que cette proposition a finalement été rejetée. Je dois préciser qu'un responsable syndical s'était fortement investi également dans cette journée, souhaitée par le proviseur mais organisée par lui-même.

(14) On peut ainsi sans doute interpréter le fait que certains modules, contestés parfois syndicalement lors de leur mise en place, soient par la suite défendus lorsqu'ils sont remis en question.

(15) Cinq d'entre eux ont d'ailleurs institué une commission pédagogique, chargée de fédérer les projets ou de les organiser. L'idée 
d'instaurer une commission pédagogique a été évoquée dans plusieurs textes officiels. Le protocole signé en 2002 sur la revalorisation du métier de chef d'établissement parle de " conseil pédagogique à venir ".

(16) Les enquêtés estiment de deux à six demi-journées hebdomadaire par semaine, selon les cas, leur durée moyenne d'absence de l'établissement.

(17) Les chefs d'établissement réfutent d'ailleurs énergiquement une interprétation des rapports entre direction et enseignants sur le mode du conflit de classe. "Pour certains collègues, ce sera toujours le cas, il y a une espèce de lutte de classes dans l'établissement » ; « Moi, je suis pas le représentant du grand capital attaqué par le prolétariat..."

(18) Avec plus ponctuellement, quelques projets de voyage à EuroDisney!
(19) Rappelons que sur les problèmes les plus importants, Dotation horaire globale ou suppression de postes, le chef d'établissement n'a guère nonce que sur la répartition du budget. Un des moyens pour protester contre une réduction de moyens est alors de ne pas voter sa répartition ou de s'abstenir, sans que le chef d'établissement ne soit mis en cause en général, et alors qu'il déplore lui-même en général cette baisse de moyens.

20) Le SNPDEN, principal syndicat des chefs d'établissement a demandé il y a quelques temps à rencontrer le SNES pour évoquer des cas de "harcèlement » par les enseignants. Le SNES a dit découvrir le problème, dont on peut être évidemment sûr a dit découvrir le problème, dont on peut être évidemment sûr rendant l'accusation réversible (Le Goff, 2003).

\section{BIBLIOGRAPHIE}

ANDRÉo C. (2005). Déviance scolaire et ordre social. Lille: Presses universitaires du Septentrion.

BALLION R. (1986). Le lycée, une cité à construire. Paris: Hachette.

BARRÈRE A. (2002a). « Un nouvel âge du désordre scolaire: les enseignants face aux incidents ", Déviance et société, vol. $26, \mathrm{n}^{\circ} 1$, p. 3-19.

BARRÈre A. (2002b). «Pourquoi les enseignants ne travaillent-ils pas en équipe? ". Sociologie du travail, vol. $44, n^{\circ} 4$, p. $481-487$.

BLANCHET R. ; Wiener C. \& Isambert J.-P. (1999). La revalorisation du rôle des chefs d'établissement de l'enseignement secondaire. Rapport à Monsieur le ministre de l'Education nationale, de la Recherche et de la Technologie et à Madame la ministre déléguée chargée de l'enseignement scolaire. Paris : MENRT, $67 \mathrm{p}$.

Boulet M.-H. ; Delattre J. \& Sagot M. (1996). « Travail encadré des lycéens ». Spirale $\mathrm{n}^{\circ} 33$, p. 53-71.

Bouveau P. \& RocheX J.-Y. (1997). Les ZEP, entre école et société. Paris : Hachette.

Braxmeyer N. \& Do C.-L. (2002). Le travail en commun des enseignants du second degré. Vanves [Hauts-deSeine] : Ministère de la Jeunesse, de l'Éducation nationale et de la Recherche : Direction de la programmation et du développement [Les Dossiers-MJENR ; $n^{\circ} 131$ ].

ComBAz G. (2002). « Le projet d'établissement scolaire : vers une dérive du curriculum ? Contribution à une sociologie des rapports État-école ". Revue française de pédagogie, $\mathrm{n}^{\circ} 139$, p. 7-19.

Cousın O. (1998). L'efficacité des collèges. Paris : PUF.

DEMAILly L. (2005). « Enjeux et limites de l'obligation de résultats : quelques réflexions à partir de la politique d'éducation prioritaire en France ". In C. Lessard \& P. Meirieu (dir.), L'obligation de résultats en éducation. Laval [Québec] : Presses de l'université Laval, p. 115-123.

DEMAILLY L. (1998). « La restructuration des rapports de travail dans les métiers relationnels ".Travail et emploi, $\mathrm{n}^{\circ} 76$, p. 3-13

Dejours C. (1998). Souffrance en France. Paris: Éd. du Seuil.

DEROUET J.-L. (1987). «Approches ethnographiques en sociologie de l'éducation: l'école et la communauté, l'établissement scolaire, la classe ». Revue française de pédagogie, $\mathrm{n}^{\circ} 78$, p. 153-170.

DeRouet J.-L. \& DutercQ Y. [dir.] (1998). L'établissement scolaire, entre autonomie locale et service public. Paris: ESF ; INRP.

Derouet J.-L. [dir.] (1999). L'école dans plusieurs mondes. Bruxelles ; Paris : De Boeck ; INRP.

DEROUET J.-L. (2000). « L'administration de l'éducation nationale: l'école de la République face au nouveau management public ». In A. van Zanten (dir.), L'école, l'état des savoirs. Paris : La Découverte, p. 103-112.

Dulot A. (1998). Politique d'établissement et politiques publiques. Paris : Ministère de l'Éducation nationale, de la Recherche, de la Science et de la Technologie.

DutercQ Y. (2003). "L'évolution du métier et des missions des principaux de collège ". In J.-L. Derouet (dir.), Le collège unique en questions. Paris : PUF, p. 301-311.

DUPRIEZ V. (2002). «Les établissements et leur contexte: entre tradition et transformations, que révèle l'analyse des établissements scolaires ». In C. Maroy, L'enseignement secondaire et ses enseignants. Bruxelles : De Boeck, p. 55-93.

DurAnd J.-P. (2004). La chaîne invisible. Paris : Éd. du Seuil.

DuRu-Bellat M. (2002). Les inégalités sociales à l'école. Paris : PUF.

FABRE M. (2006). «La pédagogie et les pédagogies ». In J. Beillerot \& N. Mosconi (dir.), Traité des sciences et des pratiques de l'éducation. Paris : Dunod, p. 501-512.

FRANCE : MINISTÈRE DE L'ÉducATION NATIONALE, DE L'ENSEIGNEMENT SUPÉRIEUR ET DE LA RECHERCHE (2004). Rapport de jury du concours de recrutement des personnels de direction d'établissements d'enseignement et de formation. Paris : Scéren/CNDP.

GATHER THURLER M. (2001). Innover dans l'établissement scolaire. Issy-les-Moulineaux : ESF.

Guillaume F.-R. (1997a). "Le rôle pédagogique des chefs d'établissement en fonction de leurs conceptions pédagogiques ». Éducations et formations $n^{\circ} 49$, p. 13-19.

Guillaume F.-R. (1997b). "Typologie des chefs d'établissement en fonction de leurs conceptions pédagogiques ", Éducations et formations $n^{\circ} 49$, p. 21-22. 
HousSAYE J. (1999). "Le soutien va-t-il tuer la pédagogie différenciée? ". Cahiers pédagogiques, $\mathrm{n}^{\circ} 376-377$, p. 13-15.

KERLAN A. (2003). "Parcours diversifiés: le collège unique en trompe-I'œil ? ». In J.-L. Derouet (dir.), Le collège unique en questions. Paris : PUF, p. 133-149.

KHERROUBI M. (2003). "La division du travail éducatif à l'épreuve du désordre scolaire ". In J.-L. Derouet (dir.), Le collège unique en questions. Paris : PUF, p. 327-339.

KHERROUBI M. \& RocheX J.-Y. (2004). « La recherche en éducation et les ZEP en France. 2. Apprentissages et exercice professionnel en ZEP : résultats, analyses, interprétations ", Revue française de pédagogie, $n^{\circ} 146$, p. 115-190.

LE Goff J.-P. (2003). « que veut-dire le harcèlement moral ». Le débat, $\mathrm{n}^{\circ} 123$, p. 141-161 \& $\mathrm{n}^{\circ} 124$, p. 99-116.

MAsson P. (1999). Les coulisses d'un lycée ordinaire. Paris : PUF.

MeRLE P. (2005). L'élève humilié : l'école un espace de non droit. Paris : PUF.

OBIN J-P. (1996). Les établissements scolaires entre l'éthique et la loi. Paris : Hachette.

PAYET J.-P. (1997). « Le sale boulot: division morale du travail dans un collège de banlieue ", Les annales de la recherche urbaine, $\mathrm{n}^{\circ} 75$, p. 75-89.
Pelage A. (2000). "Les transformations du rôle du chef d'établissement d'enseignement secondaire ". In A. van Zanten (dir.), L'école, l'état des savoirs. Paris : La Découverte, p. 219-228.

Pelage A. (2003). "La redéfinition du métier de chef d'établissement secondaire: changement statutaire, construction de l'engagement professionnel et épreuves pratiques ". Revue française de pédagogie, $\mathrm{n}^{\circ} 145$, p. 21-36.

RAYOu P. [coord.] (2002). Éducations et sociétés : revue internationale de sociologie de l'éducation, $\mathrm{n}^{\circ} 9$ : «À quoi sert la sociologie de l'éducation?".

Robert A. \& Mornettas J.-J. (1994). «Les professeurs aujourd'hui, le syndicalisme, la profession ». Revue française de pédagogie, $\mathrm{n}^{\circ} 109$, p. 89-105.

TARDIF M. \& LESSARD C. (1999). Le travail enseignant au quotidien. Bruxelles : De Boeck.

Terrall J.-P. (2002). De l'inégalité scolaire. Paris: La Dispute.

van Zanten A. (2001). L'école de la périphérie. Paris: La Découverte.

van Zanten A. ; Grospiron M.-F. ; Kherroubi M. \& Robert A. (2002). Quand l'école se mobilise. Paris : La Dispute. 